Supplementary Information

\title{
Interaction of Genetically Encoded Photosensitizers with Scintillating Nanoparticles for X-Ray Activated Photodynamic Therapy
}

\author{
Mariana C. Micheletto, Éder J. Guidelli, Antonio J. Costa-Filho* \\ Departamento de Física, Faculdade de Filosofia, Ciências e Letras de Ribeirão Preto, \\ Universidade de São Paulo, Ribeirão Preto, SP, Brazil
}

* Corresponding author: ajcosta@usp.br 
Table S1: Extinction coefficient at $280 \mathrm{~nm}$ wavelength, and theoretical pI of GFP, KillerOrange and KillerRed.

\begin{tabular}{ccc}
\hline Protein & $\varepsilon_{280 \mathrm{~nm}}\left(\mathrm{g. \textrm {I } ^ { - 1 }}\right)$ & $\mathrm{pl}$ \\
\hline GFP & 0.765 & 6.95 \\
KillerOrange & 0.476 & 6.09 \\
KillerRed & 0.308 & 7.07 \\
\hline
\end{tabular}


Table S2: Estimation of CFU concentration.

Estimation of colony-forming units at zero growth time after the different irradiation conditions of $E$. coli crops in the presence of the KR-LaF complex. The errors are the standard deviations obtained by counting colonies in four different plaques of each sample.

\begin{tabular}{cc}
\hline Condition & Bacterial concentration (unit/ml) \\
\hline Dark & $18.915 \pm 0.004$ \\
UV-A 1.5 h & $18.674 \pm 0.005$ \\
UV-B 3.0 h & $18.783 \pm 0.006$ \\
X-ray 25 Gy & $18.1 \pm 0.1$ \\
X-ray 50 Gy & $17.01 \pm 0.06$ \\
\hline
\end{tabular}


a

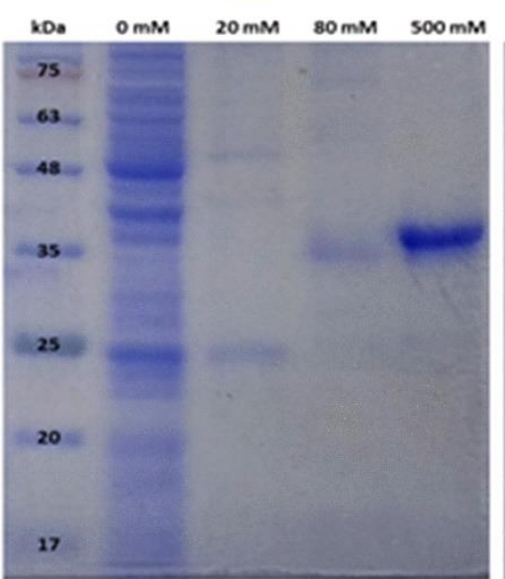

b

killerorange C

C KillerRed
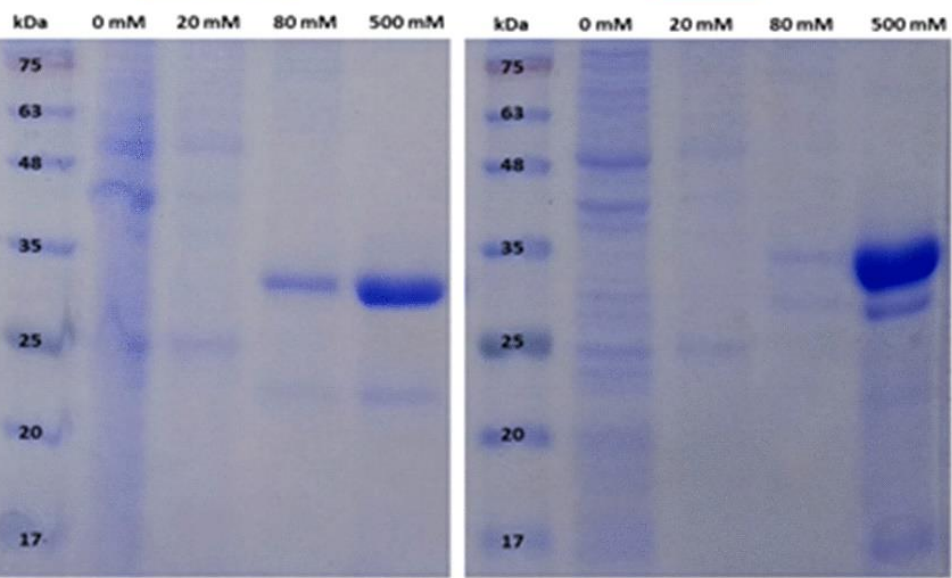

Figure S1: SDS-PAGE.

GFP proteins (a), KillerOrange (b) and KillerRed (c) purification in liquid chromatography upon an increased imidazole concentration gradient. Protein elution occurred when a wash step of 500 mM Imidazole was used. 

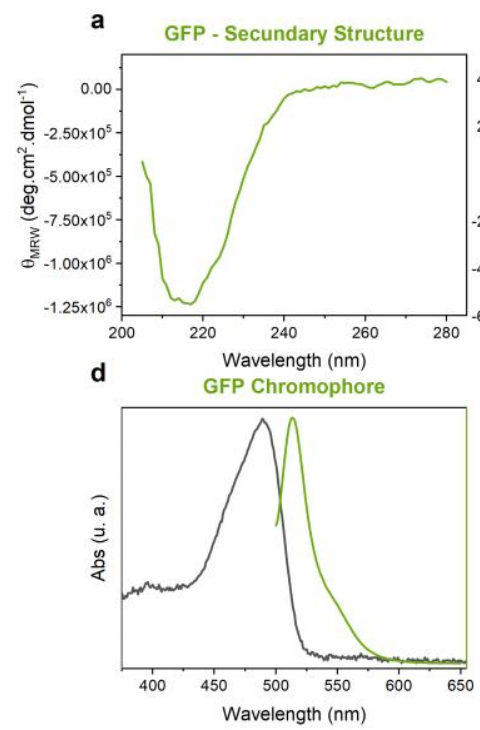

b KillerOrange - Secundary Structure

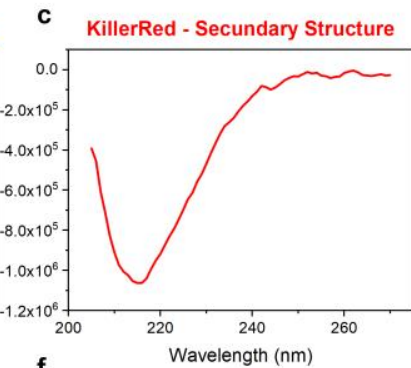

e

Wavelength $(\mathrm{nm})$
KillerOrange Chromophore
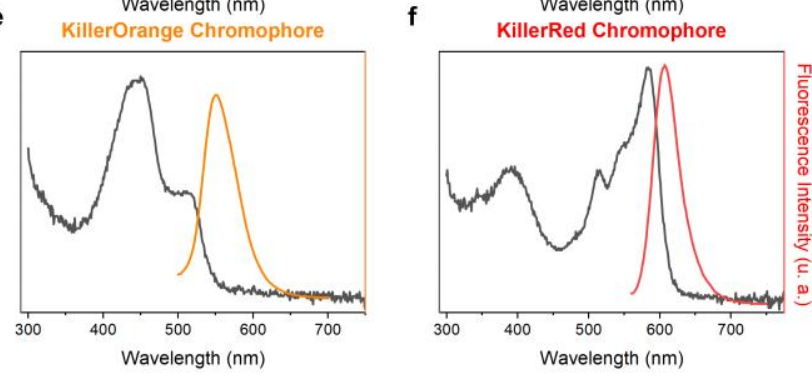

Figure S2: Structural and functional characterization.

Monitoring secondary structure of (a) eGFP, (b) KO and (c) KR by CD. Absorption (black lines) and Fluorescence (color lines) spectra of (d) eGFP, (e) KO and (f) KR. 

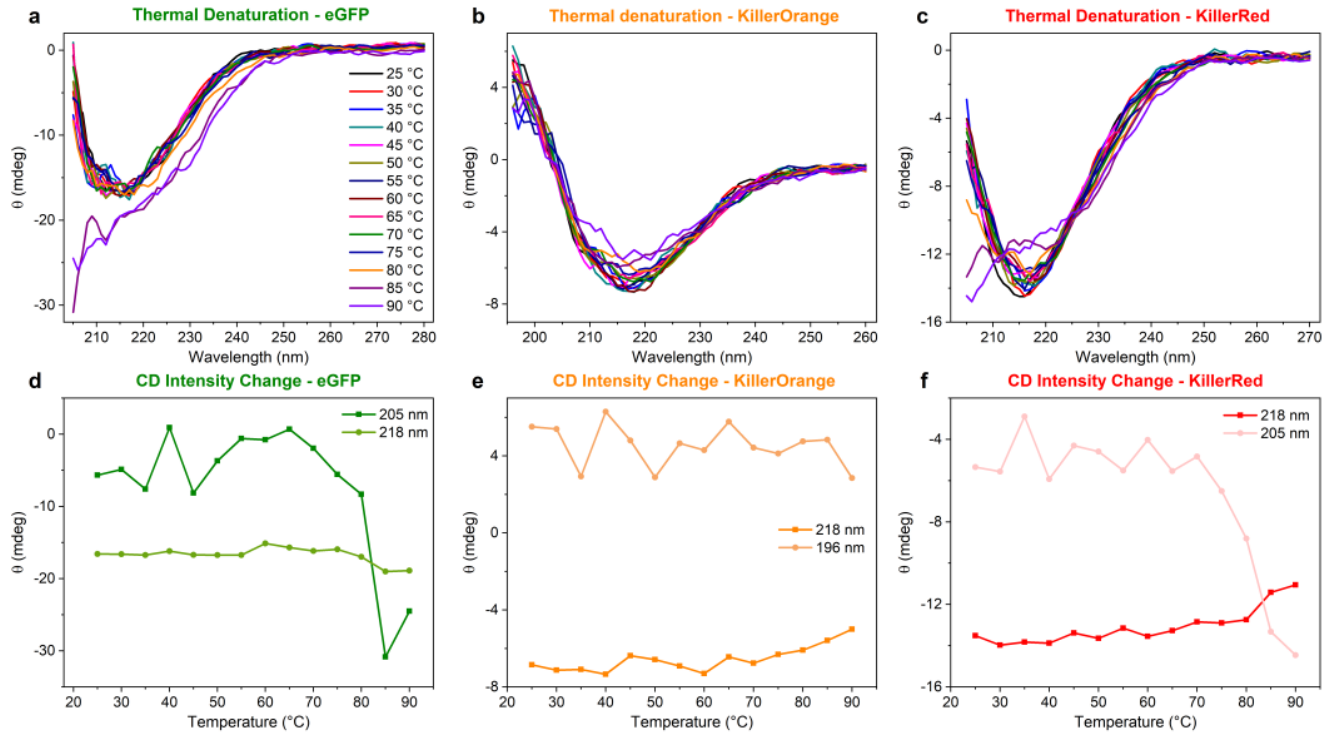

Figure S3: Secondary Structure Thermal Stability.

Monitoring secondary structure of (a) eGFP, (b) KO and (c) KR by CD and, CD intensity variation at 205 and $218 \mathrm{~nm}$ of (d) eGFP, (e) KO and (f) KR upon temperature gradient. 

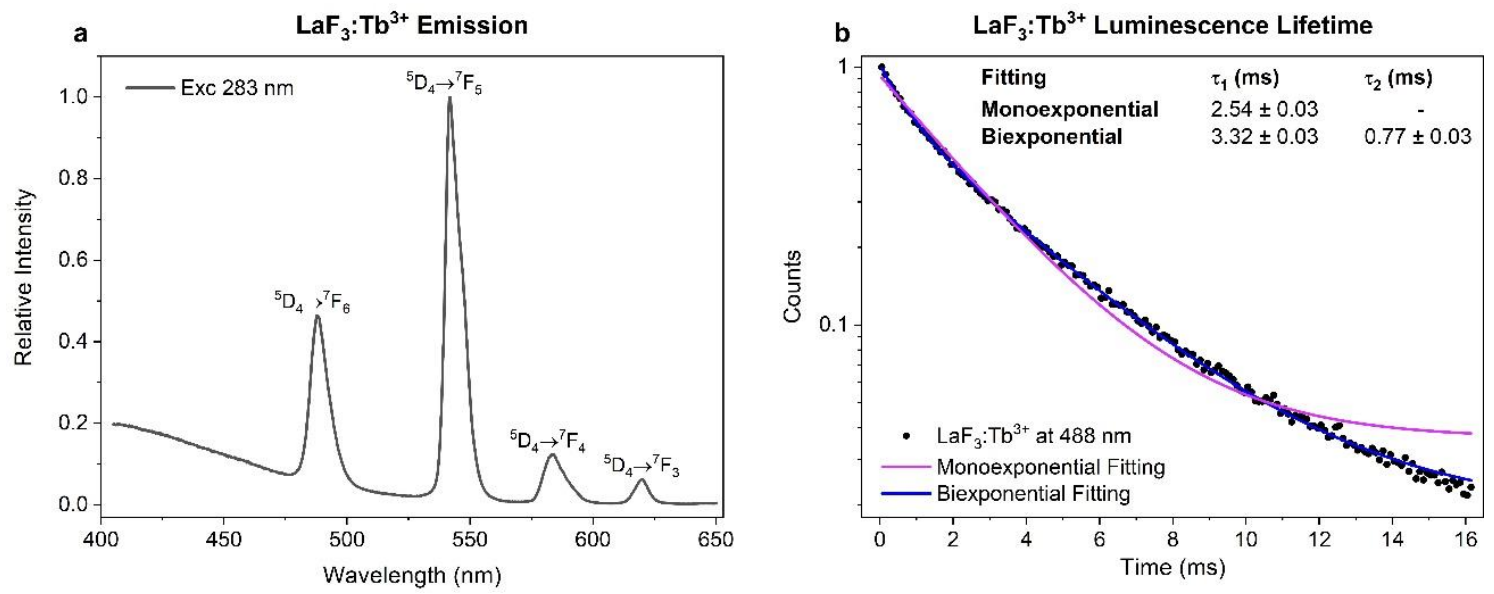

Figure S4: $\mathrm{LaF}_{3}: \mathrm{Tb}^{3+}$ spectral profile and luminescent decay.

a) PL spectrum of $\mathrm{LaF}_{3}: \mathrm{Tb}^{3+}$. b) Luminescence lifetimes recorded at $488 \mathrm{~nm}$ for $\mathrm{LaF}_{3}: \mathrm{Tb}^{3+}$ in water and mono and biexponential fitting. The errors were determined from the curve fitting. 

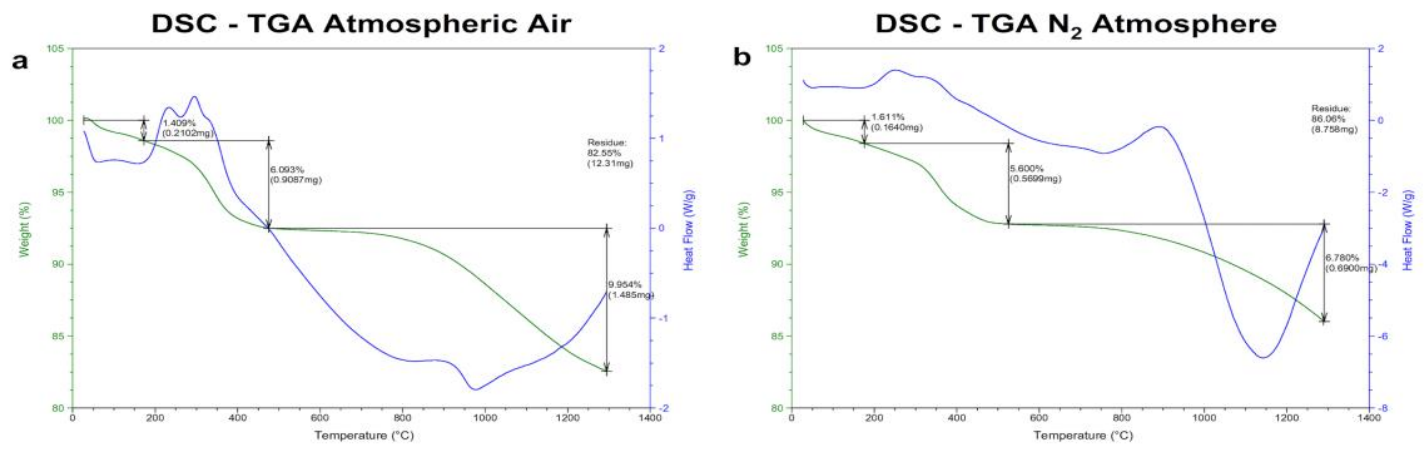

Figure S5: $\mathrm{LaF}_{3}: \mathrm{Tb}^{3+}$ thermal decomposition in air and inert atmosphere.

TGA curve of the $\mathrm{LaF}_{3}: \mathrm{Tb}^{3+}$ nanoparticles in (a) atmospheric air and (b) $\mathrm{N}_{2}$ atmosphere. 

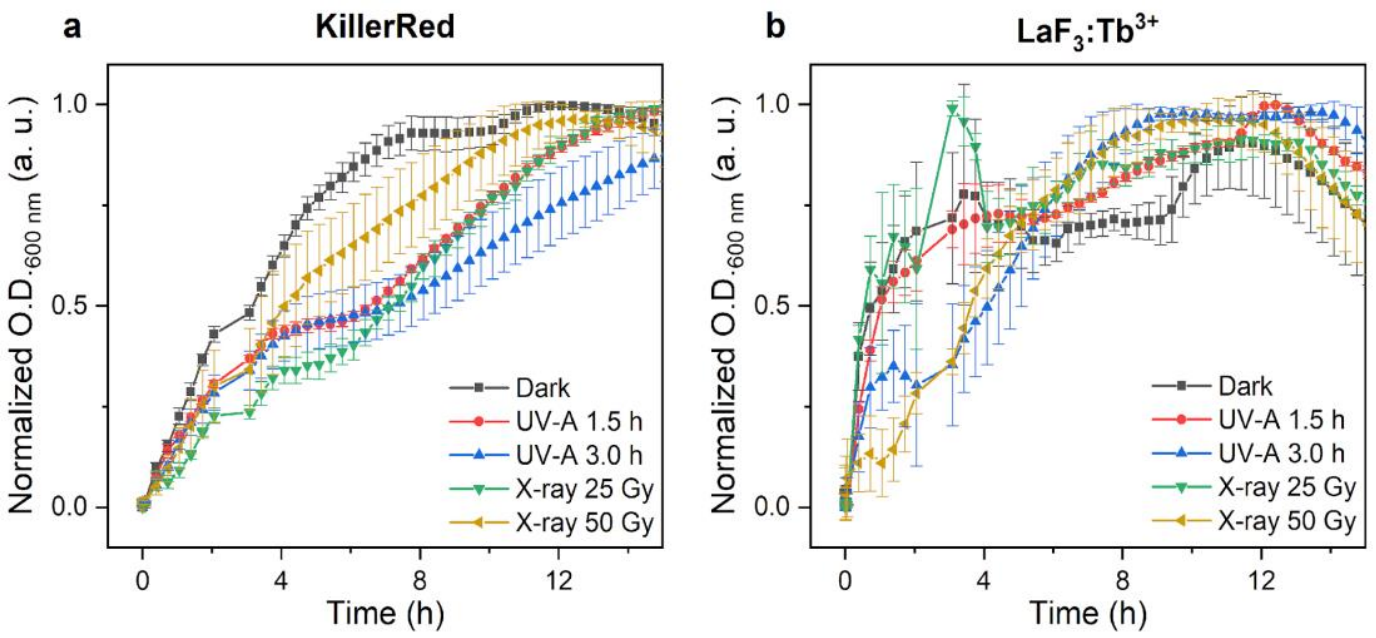

Figure S6: Monitoring E. coli growth upon UV-A and X-ray Irradiation.

Growth course of E. coli Rosetta (DE3) in LB media upon UV-A and X-ray irradiation in the presence of (a) KillerRed and (b) $\mathrm{LaF}_{3}: \mathrm{Tb}^{3+}$. The error bars are the standard deviations obtained by measuring four different samples in each condition. 